\title{
Molecular Docking Studies of Antimalarial Drugs for Malaria
}

\author{
Nutan Prakash*, Shivani Patel, Nilkanth J. Faldu, Ravi Ranjan and DVN Sudheer \\ Lecturer, Department of Biotechnology, Shree M. \& N. Virani Science College Rajkot, India
}

\begin{abstract}
Malaria is the most important parasitic disease in humans, with transmission occurring in over 100 countries with a population of three billion people. It is caused by protozoan parasites of the genus Plasmodium. These parasites are transmitted from one person to another by the female anopheles mosquito. Proguanil is a prophylactic antimalarial drug, it stops the malaria parasite, Plasmodium falciparum and Plasmodium vivax, from reproducing once it is in the red blood cells. It does this by inhibiting the enzyme, dihydrofolate reductase. The side effects of these drugs make the need for the necessity of new improved drugs Conformational analysis and geometry optimization of Proguanil was performed using Argus Lab \& Hex software. When the receptor (DHFR) was docked with the drug Proguanil the energy value obtained was (-6.59) using Argus Lab and (-174.54) using hex. The most feasible position for the drug to interact with the receptor was found to be with analog 2 having energy $-9.56 \mathrm{~K}$.cal/mole using Argus Lab and $-201.92 \mathrm{~K} . \mathrm{cal} / \mathrm{mole}$ using HEX Tool. So Proguanil Analog 2 sketched using Chemsketch is detected with more significant energy values in both softwares and probable lead molecules. Further from ADME/T properties of the Analogs is also showing the better result than available drug.
\end{abstract}

Keywords: Malaria; Dihydrofolate reductase; Proguanil; Argus lab; Hex

\section{Introduction}

Malaria is the most important parasitic disease in humans, with transmission occurring in over 100 countries with a population of three billion people. The disease causes one to two million deaths each year-equal to 150 to 300 deaths each hour (WHO, 2008; Breman, 2009). It is caused by protozoan parasites of the genus Plasmodium. The malarial parasite depends on both humans and mosquitoes to carry out its deadly cycle of life. These parasites are transmitted from one person to another by the female anopheles mosquito. Plasmodium develops in the gut of the mosquito and is passed on in the saliva of an infected insect each time it takes a new blood meal. When an infected mosquito bites a human, the parasite rapidly goes to the liver within 30 minutes. There the parasite starts reproducing rapidly in the liver. The parasites then enter into red blood cells and reproduce there after bursting, the parasites releases out and spread in the host's blood. It is injected by another mosquito and the life cycle continues like this (http://www. mosquitobait.com/understanding.html). Malaria is complex but it is a curable and preventable disease. Lives can be saved if the disease is detected early and adequately treated. It is known what action is necessary to prevent the disease and to avoid or contain epidemics and other critical situations. The technology to prevent, monitor, diagnose and treat malaria exists (http://www.microbiologybytes. $\mathrm{com} /$ introduction/Malaria.html). Dihydrofolate reductase is a small enzyme that plays a supporting role, but an essential role, in the building of DNA and other processes. It manages the state of folate, a snaky organic molecule that shuttles carbon atoms to enzymes that need them in their reactions of particular importance, the enzyme thymidylate synthase uses these carbon atoms to build thymine bases, an essential component of DNA. After folate has released its carbon atoms, it has to be recycled. This is the job performed by dihydrofolate reductase (Dihydrofolate Reductase). Many antimalarial drugs are used to treat malaria. Different combination of drugs is given to the patients suffering from malaria. Proguanil is a prophylactic antimalarial drug, which works by stopping the malaria parasite, Plasmodium falciparum and Plasmodium vivax, from reproducing once it is in the red blood cells. It does this by inhibiting the enzyme, dihydrofolate reductase, which is involved in the reproduction of the parasite. Proguanil is a drug belongs to antimalarial drugs family \& a biguanide derivative that is converted to an active metabolite called cycloguanil pamoate. It exerts its antimalarial action by inhibiting parasitic dihydrofolate reductase enzyme. It has causal prophylactic and suppressive activity against $P$. falciparum and cures the acute infection. It is also effective in suppressing the clinical attacks of vivax malaria. Proguanil inhibits the dihydrofolate reductase of plasmodia and thereby blocks the biosynthesis of purines and pyrimidines, which are essential for DNA synthesis and cell multiplication. This leads to failure of nuclear division at the time of schizont formation in erythrocytes and liver. Common side effects include stomachache, sore throat, loss of hair, abnormal bruising or bleeding, fever and pale yellow urine. Sometimes, overdose of Proguanil also creates problems. Dihydrofolate reductase has been a primary target for the Proguanil drug (http://www.emedicinehealth.com/drug- atovaquone and_proguanil/article_em.htm).

The side effects of these drugs make the need for the necessity of new improved drugs hence in our research study we try to find the suitable analogues with high binding affinity, which could be a possible lead molecule. Computational Biology and bioinformatics have the potential not only of speeding up the drug discovery process thus reducing the costs, but also of changing the way drugs are designed. Rational Drug Design (RDD) helps to facilitate and speedup the drug designing process, which involves variety of methods to identify novel compounds. One such method is the docking of the drug molecule with the receptor (target). The site of drug action,

*Corresponding author: Nutan Prakash, Lecturer, Dept.Of Biotechnology, Shree M. \& N. Virani Science College Rajkot, India, Tel: 91-9016780571; E-mail: nutanp@vsc.edu.in

Received May 04, 2010; Accepted May 07, 2010; Published May 07, 2010

Citation: Prakash N, Patel S, Faldu NJ, Ranjan R, Sudheer DVN (2010) Molecular Docking Studies of Antimalarial Drugs for Malaria. J Comput Sci Syst Biol 3: 070073. doi:10.4172/jcsb.1000059

Copyright: (c) 2010 Prakash N, et al. This is an open-access article distributed under the terms of the Creative Commons Attribution License,which permits unrestricted use, distribution, and reproduction in any medium, provided the original author and source are credited. 
which is ultimately responsible for the pharmaceutical effect, is a receptor (Richon, 1994). In the field of molecular modeling, docking is a method which predicts the preferred orientation of one molecule to a second when bound to each other to form a stable complex. Knowledge of the preferred orientation in turn may be used to predict the strength of association or binding affinity between two molecules. Docking is frequently used to predict the binding orientation of small molecule drug candidates to their protein targets in order to in turn predict the affinity and activity of the small molecule.

\section{Materials and Materials}

For our present study we used biological databases like PubChem, Drug Bank, PDB (Protein Data Bank) and software's like Hex, Arguslab, molinspiration, FROG (Leite et al., 2007), ADME Tox. Drug Bank is a unique Bioinformatics/Cheminformatics resource that combines detailed drug (i.e. chemical) data with comprehensive drug target (i.e. protein). Each Drug Card entry contains greater than 80 data fields with half of the information being devoted to drug/ chemical data and theother half devoted to drug target or protein
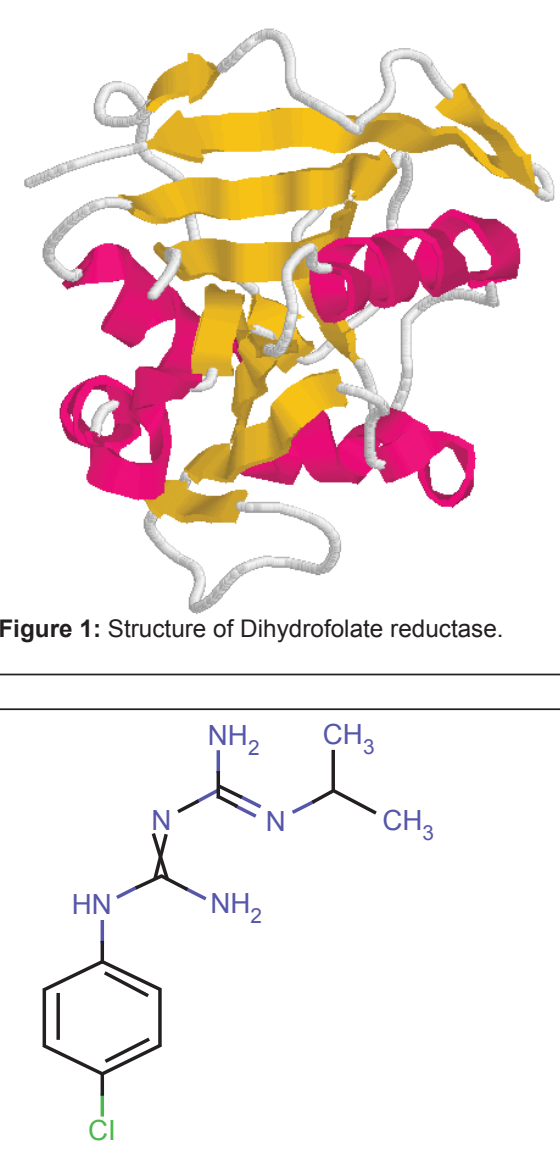

Figure 2: Structure of Proguanil.<smiles>CCN=C(N)NC(N)Nc1ccc(Cl)cc1</smiles>

Figure 3: Analog 1.

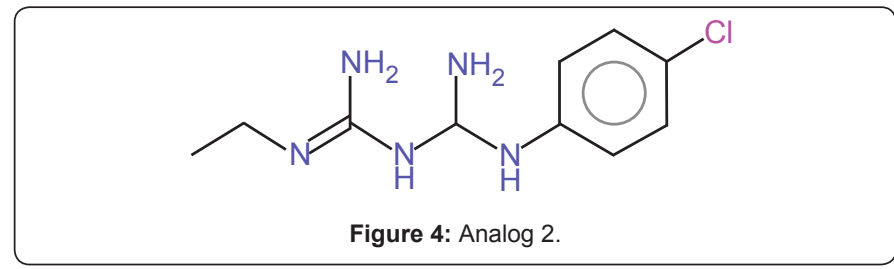

data (Drug Bank, 2006).The PDB (Protein Data Bank) is the single worldwide archive of Structural data of Biological macromolecules, established in Brookhaven National Laboratories (BNL) in 1971(The Protein Data Bank, 2000). It contains Structural information of the macromolecules determined by X-ray crystallographic, NMRmethods etc. Molinspiration is an independent research organization focused on development and application of modern cheminformatics techniques, especially in connection with the internet. Arguslab offers quite good on-screen molecule-building facilities, with a moderate library of useful molecules. It is a free molecular modeling package that runs under Windows (Thompson, 2004). Hex is an Interactive Molecular Graphics Program for calculating and displaying feasible acids and small bimolecular. The program reads in molecular coordinate files and interactively displays the molecule on the screen in variety of representations and color schemes. RASMOL [Raster Display of Molecules] is a molecular graphics program intended for the structural visualization ofproteins, nucleic acids and small biomolecules. The program reads in molecular coordinate files and interactively displaysthe molecule on the screen in variety of representations and color schemes (Horton and Stone, 1997). ADME/ TOX is an acronym in pharmacokinetics and pharmacology for absorption, distribution, metabolism, and excretion, and describes the deposition of a pharmaceutical compound within an organism. The four criteria all influence the drug levels and kinetics of drug exposure to the tissues and hence influence the performance and pharmacological activity of the compound as a drug (http://www. pharma-algorithms.com/webboxes/).

\section{Methodology}

Bioinformatics is seen as an emerging field with the potential to significantly improve how drugs are found, brought to the clinical trials and eventually released to the marketplace. Protein target were downloaded from database Protein Data Bank (PDB). 1MVT is the PDB ID of the target protein. The resolution of the protein is $1.80 \mathrm{~A}$. The target protein was visualized through Rasmol. The following figure shows the $3-\mathrm{D}$ structure of the receptor.

Proguanil SMILES was obtained from Drug bank database. The structure of drug was visualized through molinspiration. Different analogues of Proguanil drug were generated by using the SMILES of the original drug and then modifying it. The 3D structures of the analogues were obtained by using the "Frog" 3D confirmation visualizing tool.

The docking analysis of Proguanil and its analog with Human Dihydrofolate Reductase receptor was carried by HEX and Argus lab docking software. Docking allows the scientist to virtually screen a database of compounds and predict the strongest binders based on various scoring functions. It explores ways in which two molecules, such as drugs and an enzyme Human Dihydrofolate reductase receptor fit together and dock to each other well. The molecules binding to a receptor, inhibit its function, and thus act as drug (Srivastava et al., 2008). The collection of Proguanil, its derivatives and receptor complexes were identified via docking and their relative stabilities 
were evaluated using molecular dynamics and their binding affinities, using free energy simulations.

The parameters used for the docking process via HEX docking software were

- Correlation type - Shape only

- FFT Mode - 3D fast life

- Grid Dimension - 0.6

- Receptor range - 180

- Ligand Range - 180

- Twist range -360

- Distance Range - 40

The drug and its analogues were docked with the receptor using the above parameters. All the parameters used for Arguslab docking are selected by default.

\section{Result and Discussion}

Docking results of the drug and its derivatives via Arguslab docking software reveals that the e-value of analogue 2 is (-9.35) is better as compared to that of original (-6.59). The results of docking

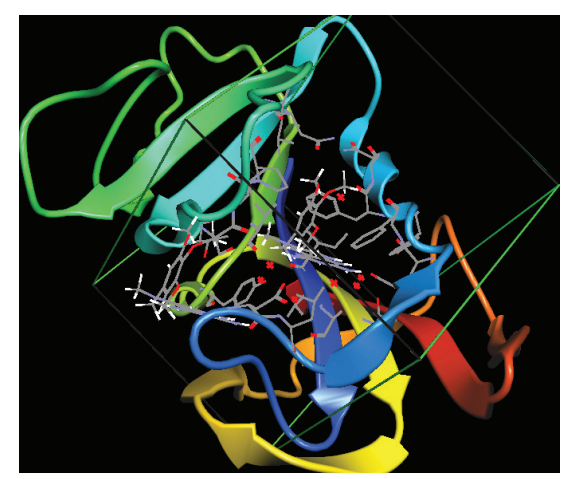

Figure 5: Docking of Analog 2 with DHFR using ArgusLab.

\begin{tabular}{|c|c|}
\hline COMPOUND & E-VALUE \\
\hline PROGUANIL & -6.59 \\
\hline ANALOG 1 & -9.24 \\
\hline ANALOG 2 & -9.56 \\
\hline
\end{tabular}

Table 1: Docking results of dihydrofolate reductase receptor with proguanil analogs using argus lab.

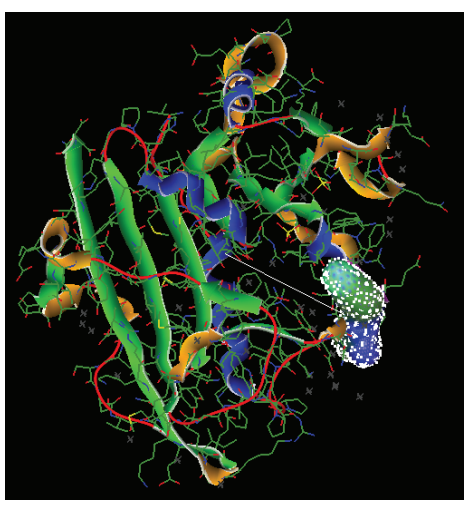

Figure 6: Docking of Analog 2 with DHFR using Hex.

\begin{tabular}{|c|c|}
\hline COMPOUND & E-VALUE \\
\hline PROGUANIL & -174.54 \\
\hline ANALOG1 & -200.81 \\
\hline ANALOG2 & $\mathbf{- 2 0 1 . 9 2}$ \\
\hline
\end{tabular}

Table 2: Docking results of dihydrofolate reductase receptor with proguanil analogs using hex.

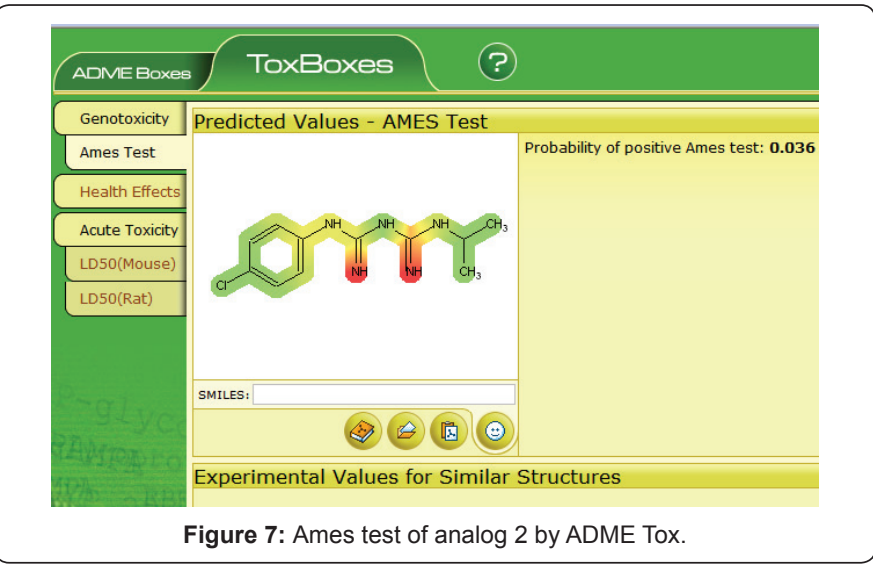

via Hex software reveal that analogue 3 has e-value better than that of original (-174.54).

Analog 2 showed an increase in the energy values of -9.56 and -201.92 respectively using Argus Lab and Hex which means the analog 2 is more compatible with the receptor than its predecessor. However, the binding site of the analog2 was similar to that of its predecessor, which means that functional groups involved were the same and by preparing the analog but the steric compatibility was increased. Here the positive Ames test indicates the probability of causing cancer. But Analog 2 is having Ames test in decrease decimal point than Proguanil which interprets that possibility of cancer is very less.

\section{Conclusion}

The Protein-Ligand interaction plays a significant role in structural based drug designing. In the present work we have taken the receptor Dihydrofolate reductase and identified the drugs that were used against Malaria. When the receptor (1MVT) was docked with the drug Proguanil the energy value obtained was (-6.59) using Argus Lab and (-174.54) using hex. When the modified drugs (Analog 2) docked against the same receptor the energy value obtained was -201.92 using Argus Lab -9.56 using HEX Tool. From this we can conclude that some of the modified drugs are better than the commercial drugs available in the market. In future research work the ADME/T (Absorption, Distribution, Metabolism, Excretion/Toxicity) properties of these compounds can be tested in wet lab and research can be proceed for clinical trials.

\section{Acknowledgment}

The support of Department of Biotechnology, Shree M.\&N. Virani Science College, Rajkot is gratefully acknowledged.

\section{References}

1. ADME/Tox WEB at http://www.pharma-algorithms.com/webboxes/.

2. Breman JG (2009) Eradicating malaria. Sci Prog 92: 101.

3. David SW, Knox C, Guo AC, Shrivastava S, Hassanali M (2006) Drug Bank: A comprehensive resource for in silico drug discovery and exploration. Nucleic Acids Res 34

4. Goodsell DS (2002) Dihydrofolate Reductase: Molecule of the Month.

5. Horton RM, Stone RJS (1997) An introduction to molecular visualisation: seeing in stereo with RasMol. Biotechniques 660 
Citation: Prakash N, Patel S, Faldu NJ, Ranjan R, Sudheer DVN (2010) Molecular Docking Studies of Antimalarial Drugs for Malaria. J Comput Sci Syst Biol 3: 070-073. doi:10.4172/jcsb.1000059

6. http://www.mosquitobait.com/understanding.html

7. http://www.microbiologybytes.com/introduction/Malaria.html

8. http://www.emedicinehealth.com/drug-atovaquone_and_proguanil/article_ em.htm

9. Leite TB, Gomes D, Miteva MA, Chomilier J, Villoutreix BO, et al. (2007) Frog: a Free Online drug 3D conformation generator. Nucleic Acids Res 35.

10. Oxfords University Press (2000) The Protein Data Bank. Nucleic Acids Res 28: 235-242.

11. Richon AB (1994) An Introduction to Molecular Modeling 1: 83.
12. Ritchie DW, Kemp GJL (2000) Protein Docking Using Spherical Polar Fourier Correlations. PROTEINS 39: 178-194.

13. Srivastava V, Kumar A, Mishra BN, Siddiqi MI (2008) Molecular docking studies on DMDP derivatives as human DHFR inhibitors. Bioinformation 4: 180-188.

14. Thompson MA (2004) Molecular docking using ArgusLab, an efficient shapebased search algorithm and the AScore scoring function ACS meeting, Philadelphia, 172, CINF 42, PA.

15. WHO (2008) World Malaria Report: Geneva Switzerland. 